\title{
Grating monochromator with ultrafast response for FLASH2 at DESY
}

Günter Brenner, Fabio Frassetto, Marion Kuhlmann, Elke Ploenjes, Luca Poletto

Günter Brenner, Fabio Frassetto, Marion Kuhlmann, Elke Ploenjes, Luca Poletto, "Grating monochromator with ultrafast response for FLASH2 at DESY," Proc. SPIE 10237, Advances in X-ray Free-Electron Lasers Instrumentation IV, 1023716 (20 June 2017); doi: 10.1117/12.2264885 


\title{
Grating monochromator with ultrafast response for FLASH2 at DESY
}

\author{
Günter Brenner ${ }^{\mathrm{a}}$, Fabio Frassetto ${ }^{\mathrm{b}}$, Marion Kuhlmann ${ }^{\mathrm{a}}$, Elke Ploenjes ${ }^{\mathrm{a}}$, Luca Poletto ${ }^{\mathrm{b}}$ \\ ${ }^{a}$ Deutsches Elektronen-Synchrotron DESY, Notkestraße 85, 22603 Hamburg, Germany; ${ }^{b}$ National \\ Research Council- Institute of Photonics and Nanotechnologies, via Trasea 7, 35131 Padova, Italy
}

\begin{abstract}
We discuss the design of grating-based monochromators for coherent ultrafast pulses in the extreme-ultraviolet. The main application of such instruments is the monochromatization of ultrafast high-order laser harmonics and freeelectron-laser pulses. We present the conditions to be fulfilled by a grating monochromator that doesn't increase the pulse duration significantly longer than the Fourier limit. A full correction of the pulse-front tilt requires the use of two gratings in a time-delay compensating configuration. The grating-monochromator configuration is applied to the design of the monochromatic beamline for FLASH2 at DESY. The monochromator has to be tunable in the $50-1000 \mathrm{eV}$ energy range with a resolving power higher than 1000 and an instrumental response shorter than $100 \mathrm{fs}$ in the whole energy range. Given the actual parameters of the FLASH2 radiation and the restrictions in the positioning of the optical elements, the tilt of the pulse-front given by a single grating would give an unacceptable temporal stretching of the pulse. This has to be corrected by a second grating in the compensated configuration. The residual distortion of the pulse-front after the second grating is well below $10 \mathrm{fs}$.
\end{abstract}

Keywords: time-delay-compensated monochromator, diffraction, variable-line-spaced grating, free-electron laser

\section{INTRODUCTION}

Free-electron laser (FEL) sources provide spatially coherent extreme-ultraviolet (XUV) and X-ray radiation with characteristics similar to the light from optical lasers, ultrashort time duration and an increase of 6-8 orders of magnitude on the peak brilliance with respect to $3^{\text {rd }}$-generation synchrotrons. There are several operating FEL facilities already open to users' experiments: FLASH in Germany (flash.desy.de), SACLA-XFEL in Japan (xfel.riken.jp), LCLS in the United States (lcls.slac.stanford.edu), FERMI in Italy (https://www.elettra.trieste.it/lightsources/fermi.html) [1-5]. Other FEL facilities are currently under development, such as the European XFEL in Germany, MAX IV in Sweden, SwissFEL in Switzerland or POLFEL in Poland (see https://www.fels-of-europe.eu).

The handling of ultrashort and ultra-intense FEL radiation is quite demanding for the optical technologies that are required to guarantee the preservation of the pulse properties [6]. In particular, due to the intrinsic multi-harmonics generation process, one of the most demanded beamline feature is the possibility to monochromatize the FEL beam even beyond the intrinsic FEL resolution, similarly with what is normally realized with synchrotron emission. Grating monochromators are already used in FEL beamlines, both at FLASH [7, 8] and LCLS [9, 10]. They give both tunability in a broad spectral range and high spectral selectivity.

The use of gratings to realize XUV monochromators for ultrafast pulses with time response on the femtosecond time scale is well established [11]. Both the single- [12] and the double-grating design [13] have been adopted. In the first case, a residual pulse-front tilt due to the diffraction has to be accepted at the output of the monochromator, that can be minimized by choosing a suitable geometry to obtain temporal responses in the range of few tens of femtoseconds in the XUV, as demonstrated in beamlines using high-order laser harmonics (HHs) [14-17]. In the second case, the design consists of a pair of gratings to compensate for the pulse-front tilt introduced by the diffraction: the first grating is demanded to perform the spectral selection on an intermediate slit while the second grating corrects for the pulse-front tilt. Double-grating instruments have been demonstrated to be very effective for HHs, with time resolution below $10 \mathrm{fs}$ [18-22]. They have also been proposed as IR-XUV beam separators for HHs [23] and as compressors for attosecond pulses $[24,25]$. Recently, double-grating configurations have been demonstrated to be effective for pulse compression to realize chirped-pulse amplification for FEL pulses in the XUV [26, 27].

In this paper, we present the design of a dedicated time-delay compensating monochromator beamline for the FLASH2 undulator line at DESY [28]. The monochromator is designed to be tunable in the $50-1000 \mathrm{eV}$ energy range with a resolving power higher than 1000 and an instrumental response shorter than $100 \mathrm{fs}$ in the whole energy range. Given the

Advances in X-ray Free-Electron Lasers Instrumentation IV, edited by Thomas Tschentscher, Luc Patthey, Proc. of SPIE Vol. 10237, 1023716 · @ 2017 SPIE · CCC code: 0277-786X/17/\$18 · doi: 10.1117/12.2264885 
actual parameters of the FLASH2 radiation (in particular its divergence) and the restrictions in the positioning of the optical elements (the minimum distance from the source), the pulse-front tilt given by a single grating would introduce an unacceptable temporal stretching of the pulse at the output of the monochromator in the picosecond time scale, as will be shown below. This is detrimental for temporal resolution and peak power of the monochromatized pulses. Therefore, the tilt has to be corrected by a second grating in the compensated configuration. The optical design originates from the variable-line-spaced (VLS) grating monochromator, that uses a plane grating with VLS grooves illuminated in converging light. A second grating is added to compensate for the tilt of the pulse-front. The residual distortion of the pulse-front after the second grating, i.e. the instrumental response of the monochromator, is well below $10 \mathrm{fs}$, as shown by the ray-tracing simulations.

The advantages of the present design are the following: 1) it minimizes the number of optical elements, since just one grating is added with respect to a standard VLS monochromator beamline; 2) it requires simple mechanical movements, of operation; 3) it fits within the design requirements for the FLASH2 experimental hall.

\section{SINGLE-GRATING MONOCHROMATORS FOR ULTRASHORT PULSES}

Single-grating monochromators for ultrafast pulses perform the spectral selection in the simplest configuration, using a grating as the dispersive element and tolerating a residual pulse-front tilt at the output. We will analyze here the conditions to have the minimum pulse-front tilt.

The grating geometry normally adopted for synchrotron and FEL radiation is the classical diffraction geometry, that is shown in Figure 1. The grating equation is $\sin \alpha-\sin \beta=m \lambda \sigma$, where $\alpha$ is the incidence angle, $\beta$ is the diffraction angle, $\sigma$ is the groove density and $m$ is the diffraction order. The angles are both taken with positive signs.

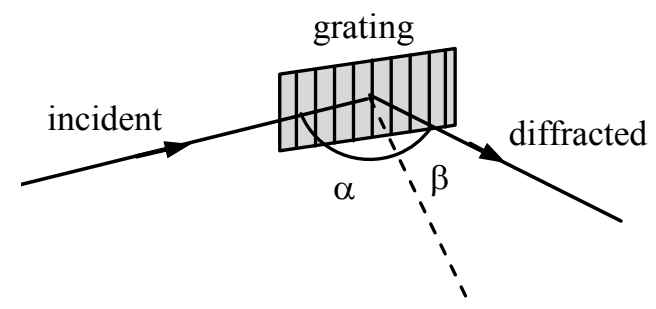

Figure 1. Reflective grating in the classical diffraction geometry.

A grating introduces a tilt of the pulse-front because of diffraction, since each ray that is diffracted by two adjacent grooves is delayed by $m \lambda$, where $\lambda$ is the wavelength. The total tilt of the pulse-front, i.e., the difference in the optical paths of the diffracted beam from the source to the image, is $|m| \lambda \mathrm{N}$, where $\mathrm{N}$ is the number of the illuminated grooves. For example, a $200 \mathrm{gr} / \mathrm{mm}$ grating illuminated by radiation at $20 \mathrm{~nm}$ over a surface of $10 \mathrm{~mm}$ gives a tilt of $40 \mu \mathrm{m}$, i.e. $130 \mathrm{fs}$. Therefore the pulse-front tilt depends on the number of grooves involved in the diffraction process.

Once the required resolution $\boldsymbol{R}=\lambda / \Delta \lambda$ at the output of the monochromator has been defined, the Rayleigh criterion states that the minimum number of grooves $\mathrm{N}_{\min }$ that have to be involved in the diffraction to support such a resolution is $|m| \mathrm{N}_{\min }=\lambda / \Delta \lambda$. The corresponding half-width variation of the optical paths at the grating output is $\Delta \mathrm{OP}_{\min } \cong 1 / 2|m| \lambda \mathrm{N}_{\min }=1 / 2 \lambda^{2} / \Delta \lambda$. It follows that the diffraction from a grating gives a lower limit for the pulse-front tilt $\Delta \tau_{\mathrm{G}, \min }$ given by

$$
\Delta \tau_{G, \min } \cong \frac{0.5}{c} \frac{\lambda^{2}}{\Delta \lambda}
$$

where $\Delta \lambda$ is the half-height spectral width and $\mathrm{c}$ the speed of light in vacuum. This value is close to the Fourier limit, that states the minimum pulse duration for a given bandwidth is

$$
\Delta \tau \cong \frac{0.44}{c} \frac{\lambda^{2}}{\Delta \lambda}
$$

for a Gaussian pulse, where $\Delta \tau$ is the half-height pulse duration.

Therefore the single-grating design can be adopted for the monochromatization of ultrashort pulses without altering in a significant way the pulse duration beyond the Fourier limit, provided that the number of illuminated grooves times the diffracted order is equal to the actual resolution. 
In the following, we discuss the design of an ultrafast monochromator with negligible front-tilt. For simplicity, we consider the plane-grating configuration shown in Figure 2. The wavelength scanning is performed by rotating the grating around the axis tangent to its vertex and parallel to the grooves by the angle

$$
\alpha=\frac{K}{2}+\arcsin \left[\frac{m \lambda \sigma}{2 \cos (K / 2)}\right]
$$

where $\mathrm{K}$ is the subtended angle: $\mathrm{K}=\alpha+\beta$.

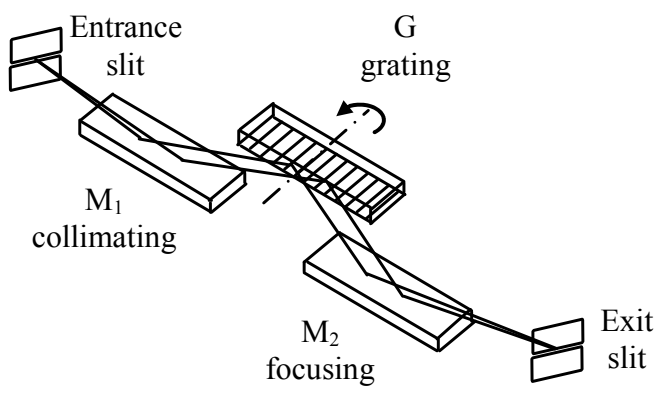

Figure 2. Plane-grating monochromator.

The resolution at the output of the monochromator depends on the spectral dispersion and on the width of the slits. Let us suppose that the width of the exit slit, $\mathrm{W}_{\text {OUT }}$, is equal to the width of the input slit, $\mathrm{W}_{\mathrm{IN}}$, as imaged at the output. This is the minimum width that has to be kept to guarantee the complete transmission of the diffracted beam. A larger width would give a poorer resolution, a smaller width would give a higher resolution although with losses in the photon flux. It results $\mathrm{W}_{\text {OUT }}=\mathrm{W}_{\text {IN }} \cos \alpha / \cos \beta q / p$, where $\cos \alpha / \cos \beta$ is the grating anamorphism and $q / p$ is the geometric magnification ( $q$ and $p$ are the input and output arms of $\mathrm{M}_{1}$ and $\mathrm{M}_{2}$, respectively). The same considerations can be applied to concavegrating monochromators, as the spherical-grating monochromator (SGM) or the toroidal-grating monochromator (TGM), where $p$ and $q$ are the grating entrance and exit arms. The resulting output bandwidth is

$$
\Delta \lambda=W_{\text {OUT }} \frac{\cos \beta}{|m| \sigma q}=W_{I N} \frac{\cos \alpha}{|m| \sigma p} .
$$

The number of illuminated grooves is $\mathrm{N}=\mathscr{D} p \sigma / \cos \alpha$, where $\mathscr{D}$ is the beam full divergence at the input. The condition $\lambda / \Delta \lambda=|m| \mathrm{N}$ to have the minimum pulse-front tilt is expressed by

$$
\mathfrak{D} W_{I N}=\lambda \text {. }
$$

The higher the divergence, the higher the pulse-front tilt given by the grating.

The condition stated in Equation 5 is very close to the relation for a diffraction-limited source: $\theta w=(4 / \pi) \lambda$ for a Gaussian beam ( $\theta$ and $w$ measured at full width). It can be concluded that the minimum pulse front-tilt that is given by a single-grating monochromator is reached when the source is diffraction-limited and the instrument is designed to give the required resolution at an exit slit that is as wide as the projection of the input source after the diffraction. If these conditions are verified, the monochromator is defined to be pulse-preserving, since the pulse-front tilt is comparable to the Fourier limit calculated for that output bandwidth.

As a test case, we present the design of a monochromator for the $20-40 \mathrm{eV}$ region with a target energy bandwidth of 50 $\mathrm{meV}$ at $30 \mathrm{eV}$. This is a typical requirement for HHs. The input beam is supposed to be Gaussian with $\mathrm{W}_{\mathrm{IN}}=100$ um and a half-width divergence $\mathscr{D}=2 \lambda\left(\pi \mathrm{W}_{\mathrm{IN}}\right)^{-1}$. The groove density is $\sigma=600 \mathrm{gr} / \mathrm{mm}$. The grating is operated at the first external order, i.e., $\beta>\alpha$, and the subtended angle is $\mathrm{K}=152^{\circ}$. The entrance arm is calculated from Equation 4 as $p=700 \mathrm{~mm}$ to give the required resolution at $30 \mathrm{eV}$. The exit arm does not influence the resolution, as soon as the width of the exit slit is chosen according to $\mathrm{W}_{\mathrm{OUT}}=\mathrm{W}_{\mathrm{IN}} \cos \alpha / \cos \beta q / p$. Let us assume an exit arm equal to the entrance arm. The resulting performances are shown in Figure 3. The pulse-front tilt is slightly higher than the Fourier limit. It is decreasing when the energy is increasing, since both beam divergence and wavelength are decreasing. It can be concluded that for a single-grating monochromator the pulse-front tilt given by the diffraction is not negligible, although it may be close to the Fourier limit for diffraction-limited sources, provided that the geometrical conditions above discussed are verified. 

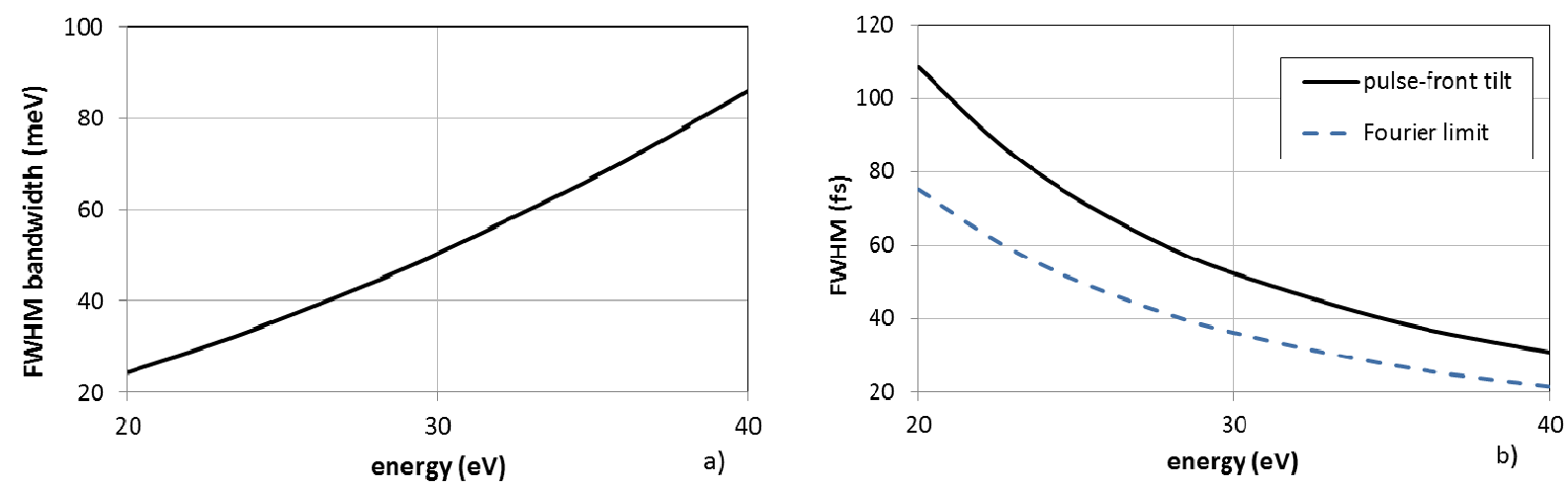

Figure 3. Ultrafast monochromator with a Gaussian diffraction-limited input source, 100 um full size. (a) Output bandwidth. (b) Temporal response of the monochromator.

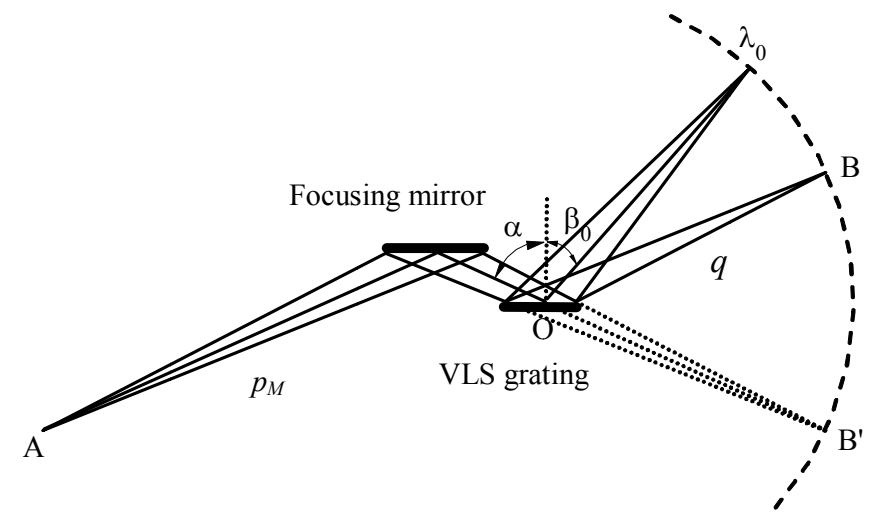

Figure 4. Flat VLS grating illuminated in converging light: schematic layout

We apply the same considerations to the variable-line-spaced (VLS) grating monochromator, that has been proposed by Hettrick and Bowyer [29] and adopted for synchrotron radiation beamlines [30] and high-order laser harmonics [31, 32]. Recently, the VLS design has been adopted also for the monochromatic beamline at LCLS (see References 9 and 10). The optical layout is shown in Figure 4. A concave mirror produces a converging beam and a VLS plane grating diffracts the radiation onto the exit slit. The variable groove spacing of the grating provides the additional free parameters to keep the focal distance almost constant as a function of the photon energy and to compensate for high-order aberrations. The VLS monochromator is also rather simple mechanically in that only two optical elements are required and the photon energy is scanned by a single rotation of the grating around an axis passing through its center following Equation (3).

The width of the output slit is equal to the projection of the input slit: $\mathrm{W}_{\text {OUT }}=\mathrm{W}_{\mathrm{IN}} \cos \alpha / \cos \beta q_{M} / p_{M}$, where $\cos \alpha / \cos \beta$ is the grating anamorphism and $q_{M} / p_{M}$ is the geometric magnification given by the concave mirror. The resulting output bandwidth is

$$
\Delta \lambda=W_{\text {OUT }} \frac{\cos \beta}{|m| \sigma q}=W_{I N} \frac{\cos \alpha}{|m| \sigma q} \frac{q_{M}}{p_{M}}
$$

where $q$ is the grating arm, that is the distance between the grating and the focal plane. The number of illuminated grooves is $\mathrm{N}=2 \mathfrak{D}\left(p_{\mathrm{M}} / q_{\mathrm{M}}\right)(\sigma q) / \cos \alpha$. The condition $\lambda / \Delta \lambda=|m| \mathrm{N}$ to have the minimum pulse-front tilt is again expressed by Equation 5 . Therefore the minimum pulse front-tilt is reached when the source is diffraction-limited.

In order to illustrate the design procedure of a VLS monochromator for FELs and to find the limiting performances, we discuss a monochromator for the $50-100 \mathrm{eV}$ region with a target energy bandwidth of $30 \mathrm{meV}$ at $75 \mathrm{eV}$, i.e., $\boldsymbol{R}=2500$. The monochromator is operated without an input slit, having as the entrance slit the FEL source itself. The source is assumed to be Gaussian with $\mathrm{S}=0.4 \mathrm{~mm}$ and half-width divergence $\mathscr{D}=2 \lambda(\mathrm{S} \pi)^{-1}$. The first mirror is assumed to be placed $70 \mathrm{~m}$ from the source, that is a typical distance for FEL beamlines. Once the grating groove density and the subtended angle (that are typically defined to maximize the grating efficiency) have been fixed, the remaining 
parameters to be chosen to fulfill the required resolution are the grating arm and the mirror-to-grating distance. The combination of these two parameters is chosen in order to obtain a width of the exit slit that is usable, typically in the 100 um range. A slit much wider than 100 um gives a size of the output source from the monochromator that is too large to be finally refocused on a micrometric spot size, as often required. A slit that is much narrower than 100 um is very demanding in terms of optical and mechanical performances of the monochromator, since it requires a very large demagnification of the source and a very careful optical and mechanical design to guarantee the required stability at the output. The design parameters to fit the requirements are resumed in Table 1. The performances are shown in Figure 5. The pulse-front tilt is decreasing when the energy is increasing, being anyway slightly higher than the Fourier limit. Obviously, if the divergence of the source is higher than the diffraction limit, also the pulse-front tilt is higher. The actual values are strongly dependent from the expected characteristics of the source.

In the following we will apply the single-grating configuration to the design of a monochromatic beamline for FLASH2.
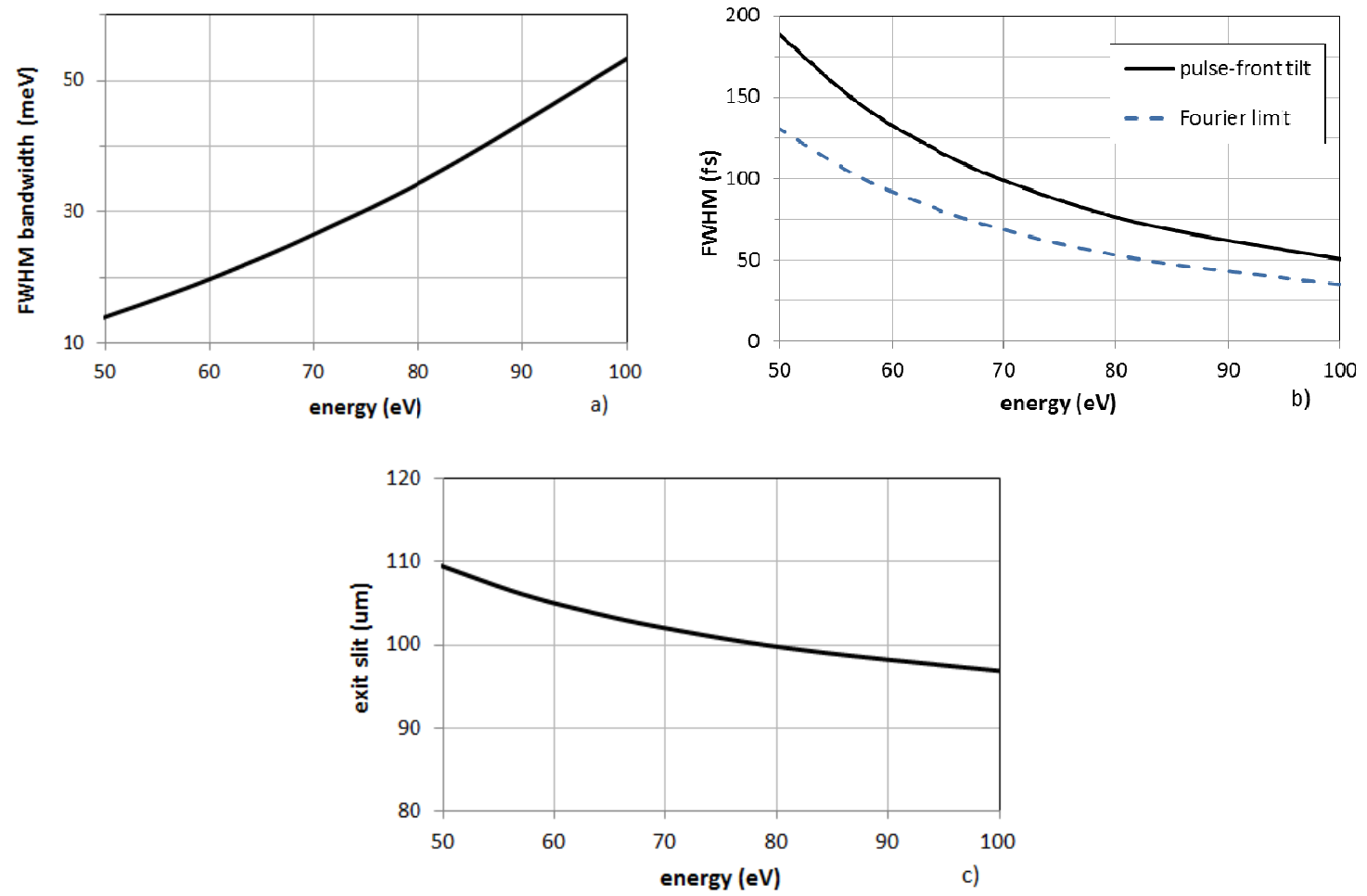

Figure 5. VLS plane-grating monochromator with a Gaussian diffraction-limited input source, 400 um full size. (a) Output bandwidth. (b) Temporal response. (c) Exit slit.

Table 1. Parameters of the VLS plane-grating monochromator for a diffraction-limited FEL source.

\begin{tabular}{|l|l|}
\hline Source size & $400 \mathrm{um}$, diffraction-limited Gaussian source \\
\hline Spectral range & $50-100 \mathrm{eV}$ \\
\hline Bandwidth & $30 \mathrm{meV}$ at $75 \mathrm{eV}$ \\
\hline Source-to-mirror distance & $70 \mathrm{~m}$ \\
\hline Mirror-to-grating distance & $7.0 \mathrm{~m}$ \\
\hline Grating-to-slit distance & $8.0 \mathrm{~m}$ \\
\hline Grating groove density & $300 \mathrm{gr} / \mathrm{mm}$ \\
\hline Grating subtended angle & $160^{\circ}$ \\
\hline Diffraction order & -1 \\
\hline
\end{tabular}




\section{DESIGN OF A MONOCHROMATIC BEAMLINE FOR FLASH2}

The main requirements for the monochromatic beamline for FLASH2 are listed in the following:

- Spectral range 60-310 eV (4-20 nm), FLASH2 fundamental emission.

- Spectral range 250-620 eV (2-5 nm), FLASH2 harmonics.

- Spectral resolution higher than 1000 over the full spectral range.

- Time response shorter than $100 \mathrm{fs}$.

Furthermore there are some additional boundary conditions on the design:

- The beamline should give minimum lateral displacement with respect to the original propagation axis of the beam at the input, to be accommodated between two adjacent beamlines that have been already designed.

- The beamline should give minimum vertical displacement to reduce the change of the beam height. Furthermore, the output beam has to be parallel to the floor.

- The first optical element of the beamline, i.e. the first deviating mirror, is placed $\approx 70 \mathrm{~m}$ from the source.

When designing a beamline for new-generation FEL sources, there are also some major issues related to the source itself that also drive the design:

- Due to the high angular and lateral stability of the source, the monochromator works without an entrance slit, i.e., the FEL itself acts as the source point for the monochromator.

- Due to high photon flux, horizontal and vertical foci have to be kept separated to reduce the radiation density on the slit blades.

The FEL source is assumed to have a size of $200 \mathrm{um}$ (standard deviation). The source divergence is taken to be 75 urad (standard deviation) at $40 \mathrm{~nm}$ and scales as $\lambda^{3 / 4}$.

\subsection{SINGLE-GRATING MONOCHROMATOR}

Let us study the performance of a VLS grating monochromator with a single grating. The optical layout is shown in Figure 6. The FEL beam is focused by the plane-elliptical mirror M1 toward the plane VLS grating G1. The latter is illuminated in converging light and diffracts the radiation toward the slit, where the beam is monochromatized. The radiation coming out from G2 is finally focused to the sample area by two plane-elliptical mirrors in the KirkpatrickBaez configuration, M3 and M4. The additional plane mirror M2 is used in combination with mirror M3 to correct the vertical deviation of the beam and have an output beam that is parallel to the floor.

Table 2. Parameters of the VLS plane-grating monochromator for FLASH2, single-grating design.

\begin{tabular}{|l|l|}
\hline Source-to-M1 distance & $70.0 \mathrm{~m}$ \\
\hline M1-to-G1 distance & $1.0 \mathrm{~m}$ \\
\hline G1-to-slit distance & $5.0 \mathrm{~m}$ \\
\hline Grating subtended angle & $172^{\circ}$, external order \\
\hline GRATING G1 & \\
\hline Spectral range & $280-620 \mathrm{eV}$ \\
\hline Groove density & $600 \mathrm{gr} / \mathrm{mm}$ \\
\hline GRATING G2 & \\
\hline Spectral range & $120-310 \mathrm{eV}$ \\
\hline Groove density & $300 \mathrm{gr} / \mathrm{mm}$ \\
\hline GRATING G3 & \\
\hline Spectral range & $120-60 \mathrm{eV}$ \\
\hline Groove density & $100 \mathrm{gr} / \mathrm{mm}$ \\
\hline
\end{tabular}




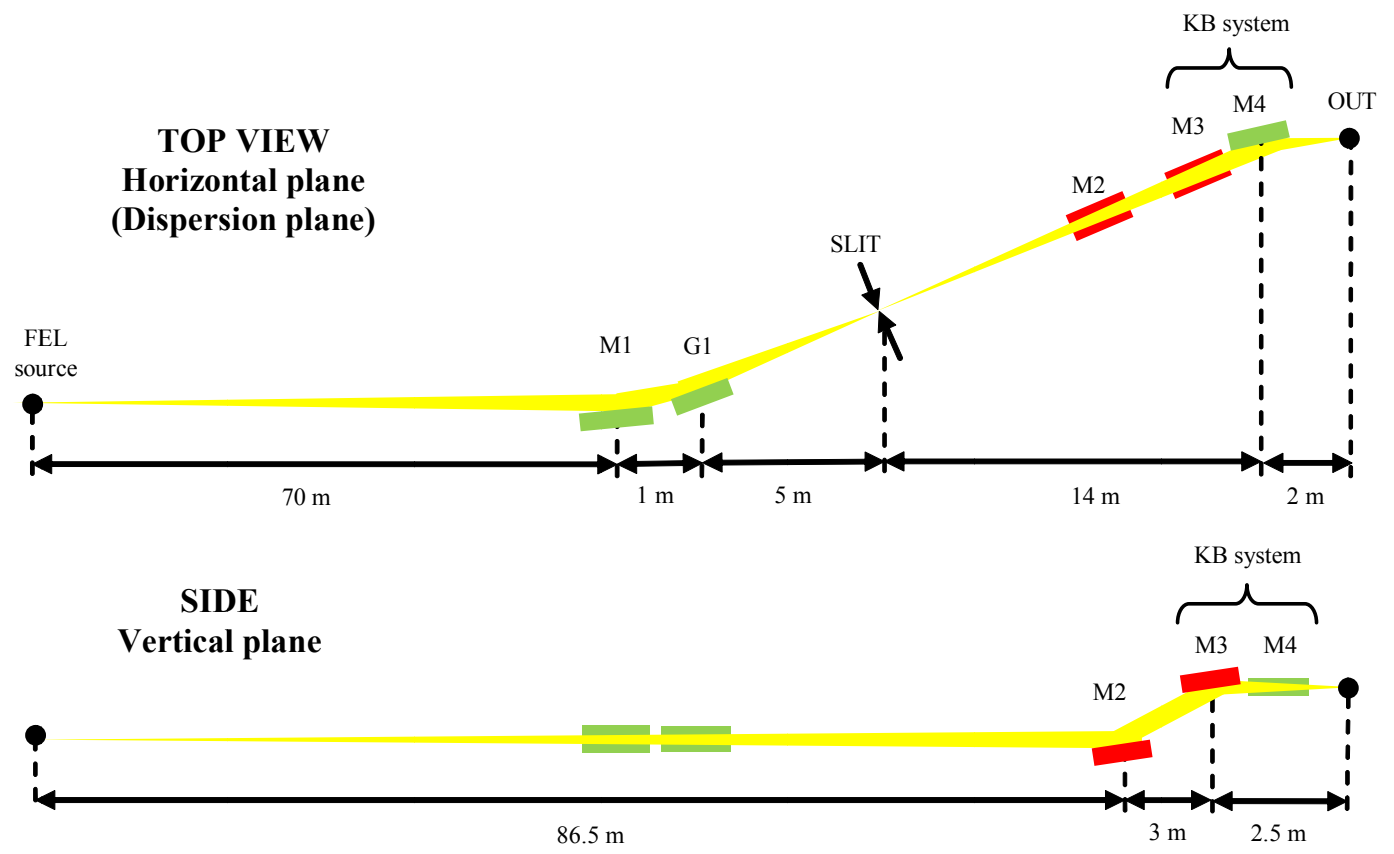

Figure 6. Layout of the VLS monochromatic beamline with a single grating. M1, G1 and M4 are focusing in the top view. M2 and M3 are focusing in the side view. The drawing is not in scale.
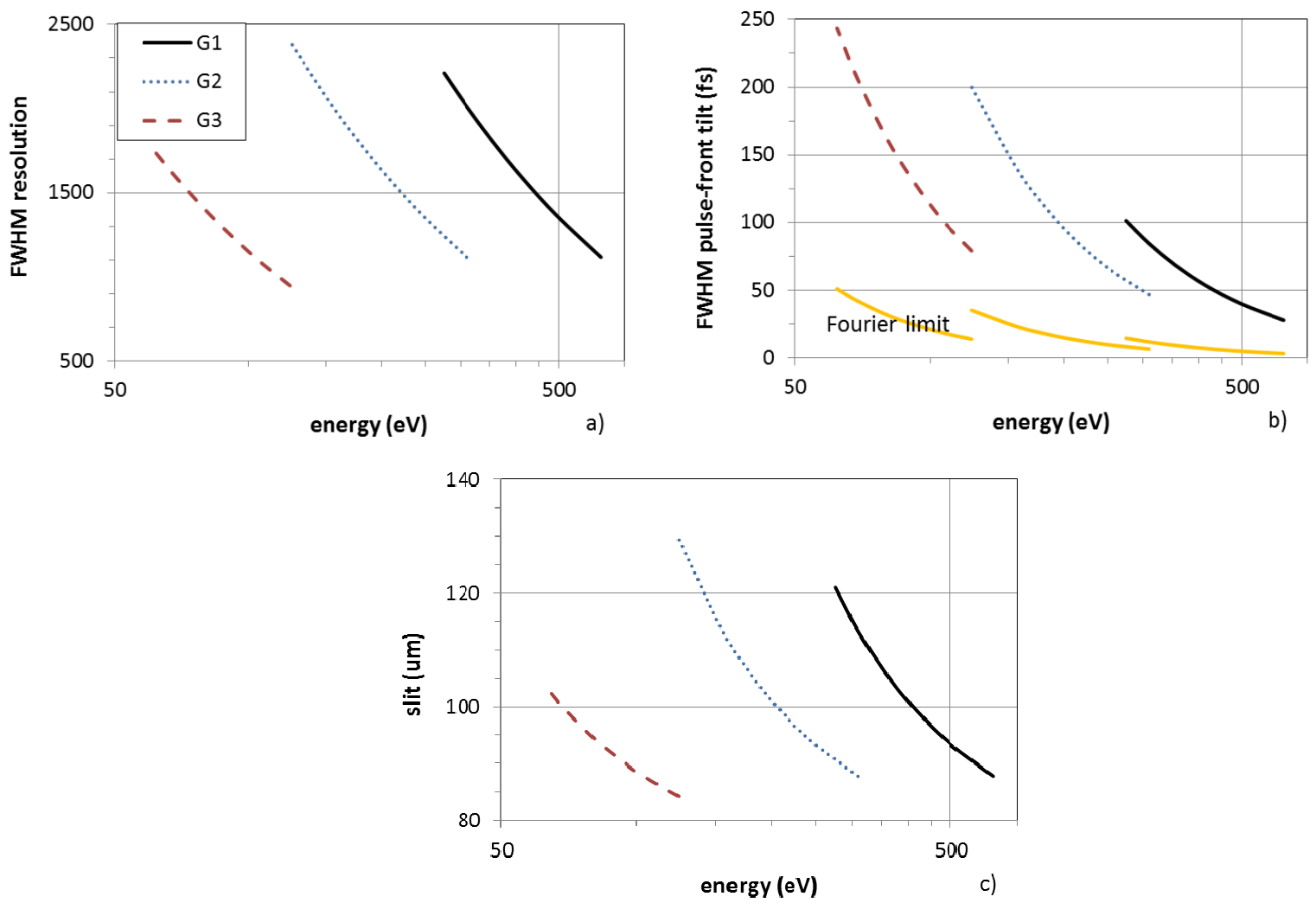

Figure 7. VLS plane-grating monochromator for FLASH2. (a) Resolution with the slit aperture shown in 7(c). (b) Temporal response. (c) Exit slit. 
The parameters are resumed in Table 2. The mirror-to-grating and grating-to-slit distances have been chosen to give the required resolution on a slit that is $\approx 100$-um wide. The full spectral range is covered by three gratings that are operated in the external order. The optical performances are resumed in Figure 7. The half-width pulse-front tilt is within the requirements for energies higher than $80 \mathrm{eV}$. At low energies, the half-width tilt can be as high as $250 \mathrm{fs}$. The pulse-front tilt is anyway well above the Fourier limit for the required resolution.

Given the FLASH2 parameters and energy range, the single-grating monochromator gives a temporal stretching that is within the requirements only for high photon energies and anyway much longer than the Fourier limit. In the following, the double-grating configuration will be presented.

\subsection{DOUBLE-GRATING MONOCHROMATOR}

In the following, the double-grating configuration will be applied to the design of the FLASH2 monochromatic beamline. A time-delay compensating grating monochromator has to adopt two gratings in compensated configuration, i.e., the second grating has to compensate for the pulse-front tilt given by the first grating, to guarantee the ultrafast response. The first grating is demanded to perform the spectral selection at the intermediate slit plane, while the second grating has to compensate for the pulse-front tilt introduced by the first grating.

The design originates from the VLS grating monochromator. The first section performs the spectral selection, while the second section has an identical VLS plane grating illuminated by the diverging light coming out from the slit and mounted in a compensated geometry. In principle, only three optical elements are required to realize a double-grating monochromator, namely the concave mirror and two VLS plane gratings, that are separated by an intermediate slit that carries out the beam monochromatization.

The beamline layout is shown in Figure 8. The FEL beam is focused by the plane-elliptical mirror M1 toward the plane VLS grating G1. The latter is illuminated in converging light and diffracts the radiation toward the intermediate slit, where the beam is monochromatized. The grating G2 has the same groove-space-variation parameters as G1 and realizes the time-delay compensating configuration. The diverging radiation coming out from G2 is finally focused to the sample area by two plane-elliptical mirrors in the Kirkpatrick-Baez configuration. Again, the additional plane mirror M2 is used in combination with mirror M3 to correct the vertical deviation of the beam and have an output beam that is parallel to the floor. The beamline consists of four mirrors and two gratings. Only one optical elements, namely the grating, is added to obtain the ultrafast response.

The optical parameters are resumed in Table 3. Two set of gratings are used: the first set is operated in the 2-7 nm (620$180 \mathrm{eV}$ ) region, where the FEL is used in the fundamental emission (above $4 \mathrm{~nm}$ ) and at higher harmonics (below $4 \mathrm{~nm}$ ); the second set is operated in the 5-20 nm (180-62 eV) region, covering the FEL fundamental.

Table 3. Parameters of the VLS plane-grating monochromator for FLASH2, double-grating design.

\begin{tabular}{|l|l|}
\hline Source-to-M1 distance & $70.0 \mathrm{~m}$ \\
\hline M1-to-G1 distance & $6.0 \mathrm{~m}$ \\
\hline G1-to-slit distance & $6.0 \mathrm{~m}$ \\
\hline Slit-to-G2 distance & $6.0 \mathrm{~m}$ \\
\hline Grating subtended angle & $174^{\circ}$ \\
\hline GRATING SET\#1 & \\
\hline Spectral range & $180-620 \mathrm{eV}$ \\
\hline Groove density & $600 \mathrm{gr} / \mathrm{mm}$ \\
\hline GRATING SET\#2 & \\
\hline Spectral range & $60-180 \mathrm{eV}$ \\
\hline Groove density & $150 \mathrm{gr} / \mathrm{mm}$ \\
\hline
\end{tabular}




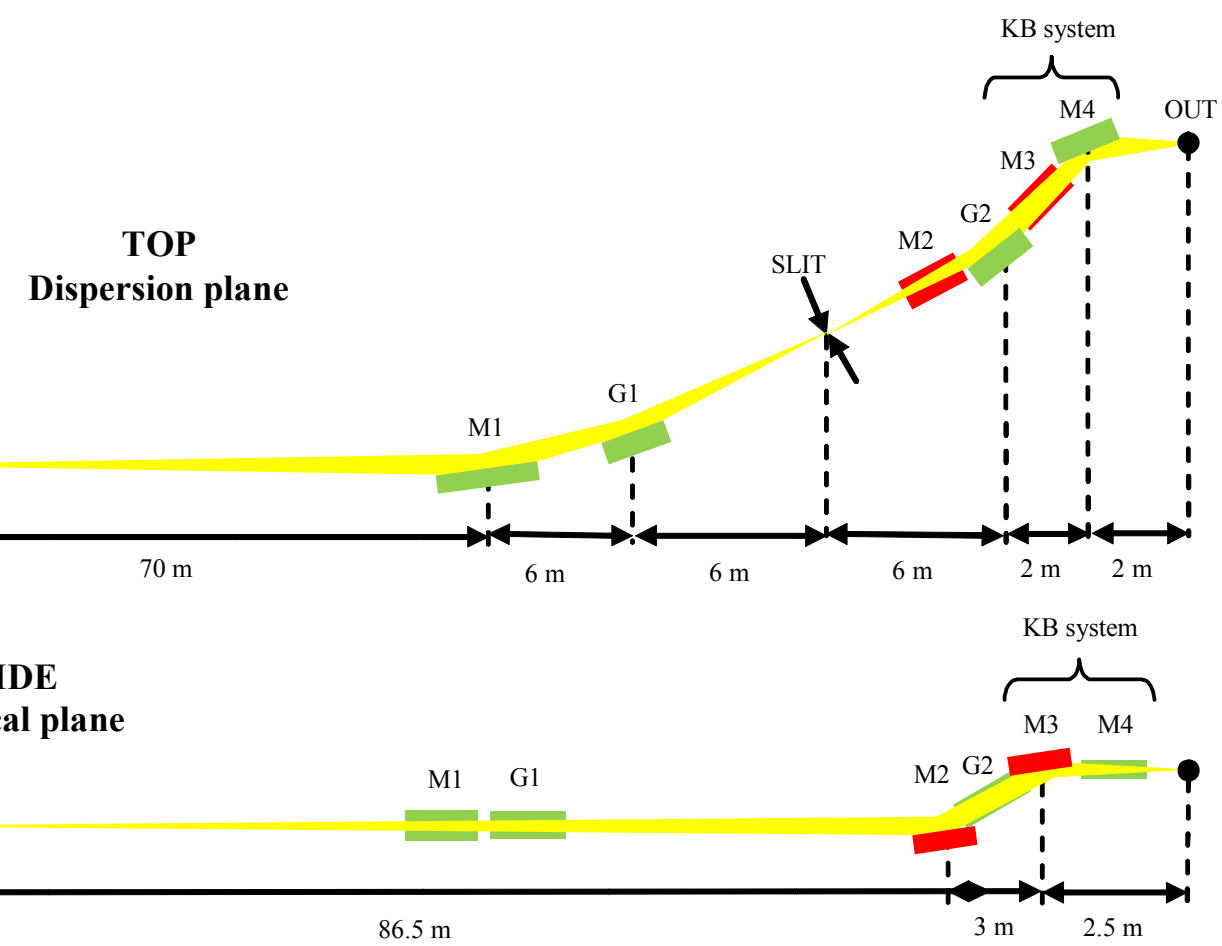

Figure 8. Schematic layout of the monochromatic beamline with the double-grating configuration. M1, G1, G2 and M4 are focusing in the top view. M2 and M3 are focusing in the side view. The drawing is not in scale.

The optical performances have been simulated by ray-tracing. The resolution on 100-um slit and the pulse-front tilt is shown in Figure 9(a). The resolution is anyway above 1000, as from the requirements. The gratings are used at I and II diffraction orders. The pulse-front tilt at the intermediate slit is shown in Figure 9(b). The tilt is related to the illuminated area on the grating, since it depends on the number of grooves involved in the diffraction. It is typically in the range 200$1000 \mathrm{fs}$, being below $200 \mathrm{fs}$ only for energies above $300 \mathrm{eV}$.
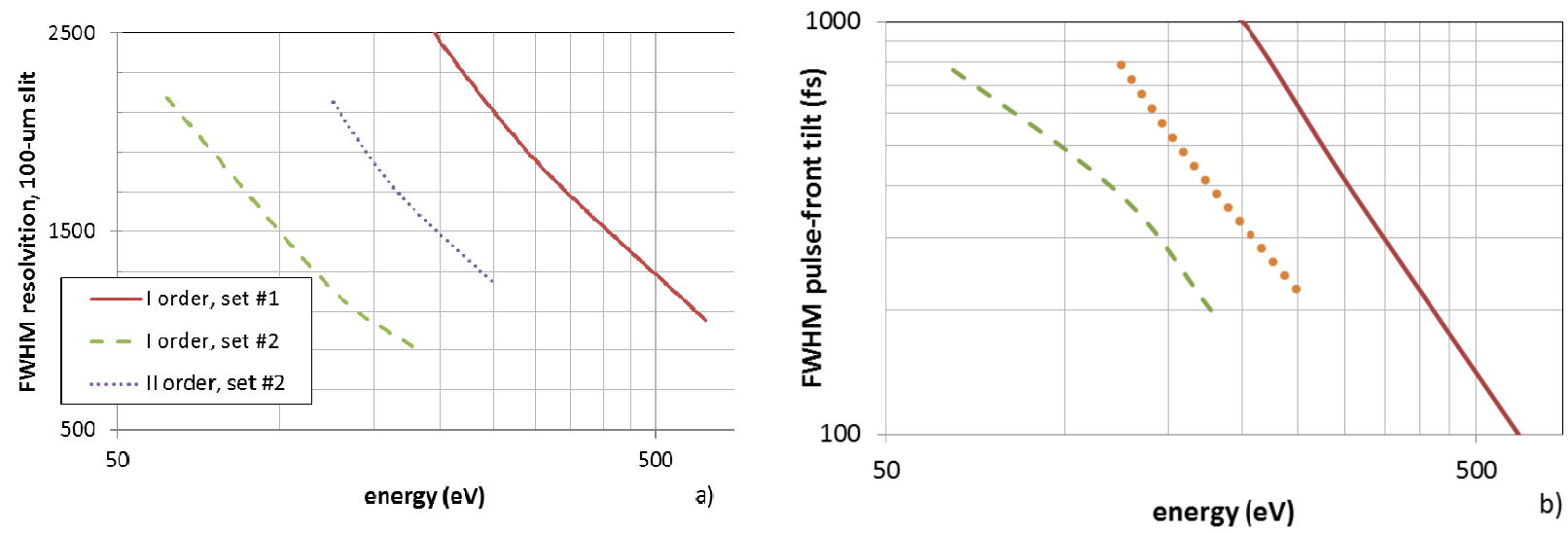

Figure 9. Performances of the double-grating monochromators. a) FWHM resolution, 100-um slit. The gratings are used at I and II diffraction order. The slit aperture is fixed at $100 \mathrm{um}$. b) pulse-front tilt at the intermediate slit.

The temporal response of the beamline is evaluated considering two different effects of the gratings on the ultrafast pulse:

- The compensation of the pulse-front tilt, i.e., all the rays emitted by the source in different directions at the same wavelength (energy) have to travel the same optical path. Ideally the compensation is perfect for a double-grating 
configuration, although aberrations may give a residual distortion of the pulse-front. This is the ultimate limit in the instrumental response of the monochromatic beamline.

- The chirp given by the two-grating configuration, i.e. different wavelengths within the bandwidth transmitted by the slit travel different paths, therefore introducing a group delay dispersion (GDD). Similarly to grating pulse shapers for the visible range, also the pair of gratings in time-delay compensated configuration may be considered as a XUV pulse shaper, capable to introduce a controllable GDD. Differently from a conventional pulse shaper, in this case the configuration is very asymmetrical, since the ratio between entrance and exit arms is about 35:1. Therefore, a nonnegligible GDD is introduced by the instrument within the bandwidth, that has been evaluated through ray-tracing.

The residual distortion of the pulse-front at the output, the chirp within the FWHM bandwidth and the Fourier limit are shown in Figure 10. The double-grating configuration is very effective in compensating for the pulse-front tilt on the slit down to a residual distortion well below $10 \mathrm{fs}$. The residual distortion of the pulse-front is the ultimate instrumental limit of any compensated configuration, that cannot be exceeded since it is due to non-corrected aberrations. This is the value to be compared with the Fourier limit and with the instrumental chirp in the given bandwidth in order to calculate the actual temporal resolution. From the data shown in the figure, the chirp introduced by the monochromator is almost the dominating factor limiting the pulse duration for energies above $70 \mathrm{eV}$. The chirp is introduced since different wavelengths travel different optical paths from the source to the final focus. It can be shown through simulations that the optical path decreases linearly with the wavelength. This forces the GDD of the double-grating monochromator to be constant and always positive. For energies lower than $70 \mathrm{eV}$, the temporal response is dominated by the Fourier limit. The spot size that is achieved at the output of the KB system is about 12 um (horizontal) $\times 8$ um (vertical) FWHM, slightly asymmetrical and almost independent from the slit aperture in the horizontal plane. This is because the doublegrating configuration compensates also for the spectral dispersion that occurs on the slit plane, i.e. different wavelengths are focused on different points on the slit plane but they are recombined on the same point at the output after the second grating. Therefore, the width of the final image in the horizontal plane is independent from the size of the slit, as soon as the slit is larger than the size of the monochromatic image itself, since dispersion is compensated. Indeed, the width of the image in the final focus is the projection of the monochromatic spot on the slit as demagnified by M4. In the vertical plane, the height of the image is the projection of the source size as demagnified by M3.

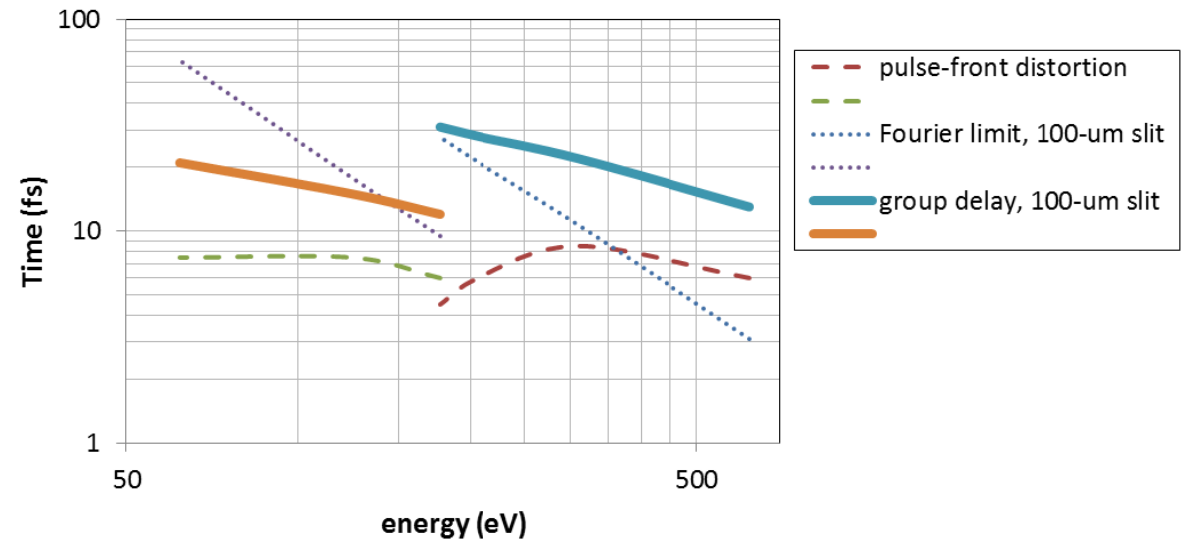

Figure 10. Temporal response of the double-grating monochromators: pulse-front residual distortion, Fourier limit with 100-um slit, group-delay within the bandwidth, 100-um slit.

\section{CONCLUSIONS}

The time response of single-grating monochromators for ultrafast pulses has been analyzed. It has been shown that timepreserving monochromators can be realized in a single-grating configuration if the number of illuminated grooves is the minimum for a given resolution, so that the pulse-front tilt is close to the Fourier limit for such a resolution.

The above considerations have been applied to the design of the monochromatic beamline for FLASH2 to be operated in the $600-60 \mathrm{eV}$ energy range. The configuration here discussed shows that a time-delay compensating monochromator can be realized in a relatively simple configuration, extending the VLS-plane-grating monochromator configuration to the use with ultrashort pulses in the extreme-ultraviolet region. The proposed configuration minimizes the number of 
optical elements, since just one grating is added with respect to a standard VLS monochromator beamline. Furthermore, it is quite simple from the opto-mechanic point-of-view: only two rotations are required to perform the spectral scan. The temporal resolution of the beamline is increased with respect to a single-grating monochromator down to few tens of femtoseconds, mainly limited by the group delay introduced by the monochromator at energies higher than $70 \mathrm{eV}$ and by the Fourier limit at the energies lower.

\section{ACKNOWLEDGMENT}

The research has been partially funded through the International Project ELI (Extreme Light Infrastructure) by the Italian Ministry for Education and Research (MIUR). LP would like to acknowledge the Stephenson Distinguished Visitor Programme (DESY Photon Science), Year 2016, for supporting the project.

\section{REFERENCES}

[1] Ackermann, W. et al., "Operation of a free-electron laser from the extreme ultraviolet to the water window," Nat. Photonics 1, 336-342 (2007).

[2] Emma, P. et al., "First lasing and operation of an angstrom-wavelength free-electron laser," Nat. Photonics 4, 66416647 (2010).

[3] Ishikawa, T. et al., "A compact X-ray free-electron laser emitting in the sub-angstrom region," Nat. Photonics 6, 540-544 (2012).

[4] Amann, J. et al., "Demonstration of self-seeding in a hard-X-ray free-electron laser," Nat. Photonics 6, 693-698 (2012).

[5] Allaria, E. et al., "Highly coherent and stable pulses from the FERMI seeded free-electron laser in the extreme ultraviolet," Nat. Photonics 6, 699-704 (2012).

[6] Canova, F. and Poletto, L., [Optical Technologies for Extreme-Ultraviolet and Soft X-ray Coherent Sources], Springer, New York (2015).

[7] Martins, M., Wellhöfer, M., Hoeft, J.T., Wurth, W., Feldhaus, J. and Follath, R., "Monochromator beamline for FLASH," Rev. Sci. Inst. 77, 115108 (2006).

[8] Guerasimova, N., Dziarzhytski, S., Feldhaus, J., "The monochromator beamline at FLASH: performance, capabilities and upgrade plans ," J. Mod. Opt. 58, 1480 (2011).

[9] Heimann, P., Krupin, O., Schlotter, W.F., Turner, J., Krzywinski, J., Sorgenfrei, F., Messerschmidt, M., Bernstein, D., Chalupský, J., Hájková, V., Hau-Riege, S., Holmes, M., Juha, L., Kelez, N., Lüning, J., Nordlund, D., Fernandez Perea, M., Scherz, A., Soufli, R., Wurth, W. Rowen, M., "Linac Coherent Light Source soft X-ray materials science instrument optical design and monochromator commissioning," Rev. Sci. Inst. 82, 093104 (2011).

[10] Schlotter, W.F., Turner, J.J., Rowen, M., Heimann, P., Holmes, M., Krupin, O., Messerschmidt, M., Moeller, S., Krzywinski, J., Soufli, R., Fernández-Perea, M., Kelez, N., Lee, S., Coffee, R., Hays, G., Beye, M., Gerken, N., Sorgenfrei, F., Hau-Riege, S., Juha, L., Chalupsky, J., Hajkova, V., Mancuso, A.P., Singer, A., Yefanov, O., Vartanyants, I.A., Cadenazzi, G., Abbey, B., Nugent, K.A., Sinn, H., Lüning, J., Schaffert, S., Eisebitt, S., Lee, W.S., Scherz, A., Nilsson, A.R. and Wurth, W., "The soft x-ray instrument for materials studies at the linac coherent light source x-ray free-electron laser,” Rev. Sci. Inst. 83, 043107 (2012).

[11] Poletto, L., Frassetto, F. and Villoresi, P., "Ultrafast Grating Instruments in the Extreme Ultraviolet," J. Sel. Top. Quant. Electron. 18, 467-478 (2012).

[12] Poletto, L. and Frassetto, F., "Time-preserving monochromators for ultrafast extreme-ultraviolet pulses," Appl. Opt. 49, 5465-5473 (2010).

[13] Poletto, L. and Villoresi, P., "Time-compensated monochromator in the off-plane mount for extreme-ultraviolet ultrashort pulses," Appl. Opt. 45, 8577-8585 (2006).

[14] Frassetto, F., Cacho, C., Froud, C.A., Turcu, I.C., Villoresi, P., Bryan, W.A., Springate, E. and Poletto, L., "Singlegrating monochromator for extreme-ultraviolet ultrashort pulses," Opt. Express 19, 19169-19181 (2011).

[15] Grazioli, C., Callegari, C., Ciavardini, A., Coreno, M., Frassetto, F., Gauthier, D., Golob, D., Ivanov, R., Kivimäki, A., Mahieu, B., Bučar, B., Merhar, M., Miotti, P., Poletto, L., Polo, E., Ressel, B., Spezzani, C. and De Ninno, G., "CITIUS: An infrared-extreme ultraviolet light source for fundamental and applied ultrafast science," Rev. Sci. Instrum. 85, 023104 (2014). 
[16] Poletto, L., Miotti, P., Frassetto, F., Spezzani, C., Grazioli, C., Coreno, M., Ressel, B., Gauthier, D., Ivanov, R., Ciavardini, A., de Simone, M., Stagira, S. and De Ninno, G. "Double-configuration grating monochromator for extreme-ultraviolet ultrafast pulses," Appl. Opt. 53, 5879-5888 (2014).

[17] Ojeda, J., Arrell, C.A., Grilj, J., Frassetto, F., Mewes, L., Zhang, H., van Mourik, F., Poletto, L. Chergui, M., "Harmonium: a pulse preserving source of monochromatic EUV $(30-110 \mathrm{eV})$ radiation for ultrafast photoelectron spectroscopy of liquids," Structural Dynamics 3, 023602 (2016).

[18] L. Poletto, "Time-compensated grazing-incidence monochromator for extreme-ultraviolet and soft X-ray high-order harmonics," Applied Physics B 78, 1013-1016 (2004).

[19] Poletto, L., Villoresi, P., Benedetti, E., Ferrari, F., Sansone, G., Stagira, S. and Nisoli, M., "Intense femtosecond extreme ultraviolet pulses by using a time-delay compensated monochromator," Opt. Lett. 32, 2897-2899 (2007).

[20] Poletto, L., Villoresi, P., Frassetto, F., Calegari, F., Ferrari, F., Lucchini, M., Sansone, G. and Nisoli, M., "Time-delay compensated monochromator for the spectral selection of extreme-ultraviolet high-order laser harmonics," Rev. Sci. Instrum. 80, 123109 (2009).

[21] Ito, M., Kataoka, Y., Okamoto, T., Yamashita, M. and Sekikawa, T., "Spatiotemporal characterization of singleorder high harmonic pulses from time-compensated toroidal-grating monochromator," Opt. Express 18, 6071-6078 (2010).

[22] Igarashi, H., Makida, A., Ito, M. and Sekikawa, T., "Pulse compression of phase-matched high harmonic pulses from a time-delay compensated monochromator," Opt. Express 20, 3725-3732 (2012).

[23] Frassetto, F., Villoresi, P. and Poletto, L., "Beam separator for high-order harmonic radiation in the 3-10 nm spectral region," J. Opt. Soc. Am. A 25, 1104-1114 (2008).

[24] Frassetto, F., Villoresi, P. and Poletto, L:, "Optical concept of a compressor for XUV pulses in the attosecond domain," Opt. Express 16, 6652-6667 (2008).

[25] Mero, M., Frassetto, F., Villoresi, P., Poletto, L. and Varjiu, K., "Compression methods for XUV attoseceond pulses," Opt. Express 19, 23420-23428 (2011).

[26] Frassetto, F. and Poletto, L., "Grating configurations to compress extreme-ultraviolet short pulses," Appl. Opt. 54, 7985-7992 (2015).

[27] Gauthier, D., Allaria, E., Coreno, M., Cudin, I., Dacasa, H., Danailov, M.B., Demidovich, A., Di Mitri, S., Diviacco, B., Ferrari, E., Finetti, P., Frassetto, F., Garzella, D., Kunzel, S., Leroux, V. Mahieu, B., Mahne, N., Meyer, M., Mazza, T., Miotti, P., Penco, G., Raimondi, L., Ribic, P.R., Richter, R., Roussel, E., Schulz, S., Sturari, L., Svetina, C., Trovò, M., Walker, P.A., Zangrando, M., Callegari, C., Fajardo, M., Poletto, L., Zeitoun, P., Giannessi, L., De Ninno, G., "Chirped pulse amplification in an extreme-ultraviolet free-electron laser," Nat. Comm. 7, 13688 (2016).

[28] Plönjes, E., Faatz, B., Feldhaus, J., Kuhlmann, M., Tiedtke, K.I., Treusch, R., "FLASH2 beamline and photon diagnostics concepts," Proceedings of FEL2013 - New York, WEPSO50, 614 (2013).

[29] Hettrick, M., and Bowyer, S., "Variable line-space gratings: new designs for use in grazing incidence spectrometers," Appl. Opt. 22, 3921 (1983).

[30] Underwood, J.H., and Koch, J.A., "High-resolution tunable spectrograph for x-ray laser linewidth measurements with a plane varied-line-spacing grating," Appl. Opt. 36, 4913 (1997).

[31] Poletto, L., Tondello, G. and Villoresi, P., "Optical design of a spectrometer-monochromator for the extremeultraviolet and soft-x-ray emission of high-order harmonics," Appl. Opt. 42, 6367 (2003).

[32] Poletto, L., Bonora, S., Pascolini, M. and Villoresi, P., "Instrumentation for analysis and utilization of estremeultraviolet and soft X-ray high-order harmonics," Rev. Sci. Instr. 75, 4413 (2004). 